\title{
Babinet's principle and the band structure of surface waves on patterned metal arrays
}

\author{
J. D. Edmunds, ${ }^{1, a)}$ M. C. Taylor, ${ }^{1}$ A. P. Hibbins, ${ }^{1}$ J. R. Sambles, ${ }^{1}$ and I. J. Youngs ${ }^{2}$ \\ ${ }^{1}$ Electromagnetic Materials Group, School of Physics, University of Exeter, Stocker Road, Exeter EX4 4QL, \\ United Kingdom \\ ${ }^{2}$ DSTL, Salisbury, SP4 OJQ, United Kingdom
}

(Received 7 January 2010; accepted 22 March 2010; published online 21 May 2010)

\begin{abstract}
The microwave response of an array of square metal patches and its complementary structure, an array of square holes, has been experimentally studied. The resonant phenomena, which yield either enhanced transmission or reflection, are attributed to the excitation of diffractively coupled surface waves. The band structure of these surface modes has been quantified for both $p$-(transverse magnetic) and $s$-(transverse electric) polarized radiation and is found to be dependent on the periodicity of the electric and magnetic fields on resonance. The results are in excellent accord with predictions from finite element method modeling and the electromagnetic form of Babinet's principle [Babinet, C. R. Acad. Sci. 4, 638 (1837)]. (C) 2010 American Institute of Physics. [doi:10.1063/1.3406145]
\end{abstract}

\section{INTRODUCTION}

Electromagnetic surface waves have been extensively studied over the last century. For example, at the beginning of the twentieth century, Zenneck ${ }^{1}$ and Sommerfeld ${ }^{2,3}$ individually developed theories proposing the propagation of electromagnetic waves along the surface of a conducting material. These surface waves are bound at the interface between two dissimilar media if the permittivity of the substrate is dominated by either a negative real part and/or a positive imaginary part. ${ }^{4}$ They are defined simply as an electromagnetic wave that propagates along the interface, and whose amplitude decays exponentially with distance into the two media either side. At the interface between two nonabsorbing media, these surface waves are known as Fano modes, ${ }^{5}$ while if one medium is absorbing they are referred to as Zenneck modes. ${ }^{6}$ The wave vector of a surface wave $k_{s w}$ for a planar interface ${ }^{7}$ separating media with complex permittivities $\varepsilon_{1}$ and $\varepsilon_{2}$ is given by

$$
k_{s w}=k_{0}\left(\frac{\varepsilon_{1} \varepsilon_{2}}{\varepsilon_{1}+\varepsilon_{2}}\right)^{1 / 2},
$$

where $k_{0}=\omega / c$.

The plasma frequency $\left(\omega_{p}\right)$ of metals typically resides in the ultraviolet region, yielding a predominantly Drude-type dielectric function dominated by a negative real part in the visible and infrared regimes for so called "plasmonic" metals such as silver and gold. Owing to their finite skin depth, their surface impedance $\left(Z_{s}=n_{2} / \varepsilon_{2}\right.$, where $n_{2}=\sqrt{\varepsilon_{2} \mu_{2}}$ is the refractive index and $\mu_{2}$ is the complex permeability of the metal, with the usual selection rules applying to the sign of the $\operatorname{root}^{8}$ ) is positive and imaginary, with a small resistive term, i.e., the surface is inductive. For $p$-polarized radiation incident through a dielectric, $\varepsilon_{1}$ a discontinuity will be induced in the component of the electric field normal to the interface, which results in a surface charge that oscillates longitudi-

\footnotetext{
${ }^{a)}$ Electronic mail: j.d.edmunds@ex.ac.uk.
}

nally when coupled to the radiation field. A bound surface wave (known as the "surface plasmon-polariton") is supported below the resonance of this surface plasmon, $\left(\omega_{s w}\right.$ $=\omega_{p} / \sqrt{1+\varepsilon_{1}}$, for a planar interface $\left.{ }^{7}\right)$, which typically occurs in the ultraviolet. Since $k_{s w}>k_{0}$ [Eq. (1)], these modes are nonradiative but can be coupled to using in-plane momentum enhancement techniques such as diffractive coupling using gratings ${ }^{7}$ or frustrated internal reflection methods. ${ }^{9,10}$ However for frequencies smaller than the scattering rate of the electrons (including the gigahertz and terahertz regimes), the dielectric constant of metals becomes dominated by its imaginary component, and the surface impedance has almost equal positive real and positive imaginary parts, i.e., the small resistance of a metal surface is accompanied by an equally (small) value of inductance. This gives loosely bound surface waves which propagate almost without attenuation at speeds close to the speed of light (i.e., $k_{0} \approx k_{s w}$ ). Their fields extend for many wavelengths into free space, and penetrate into the metal substrate by only a small fraction of the incident wavelength, therefore, these modes are commonly described as surface currents. However, as early as $1944,{ }^{11-14}$ researchers were aware that the addition of a subwavelength corrugation to the metal surface would produce an enhanced surface reactance and strongly bind the mode to the interface, even in this long-wavelength regime. For example, Barlow and Cullen ${ }^{11}$ and Cutler $^{12}$ considered a corrugated metal slab, in which each narrow groove can be regarded as a parallel-plate transverse electromagnetic transmission line. If the grooves are designed to be resonant, e.g., approximately one-quarter of a wavelength deep, then the short circuit (i.e., their closed end) at the bottom is transformed by the length of the slot into an open circuit at the open end, such that the surface impedance becomes large in magnitude. However, if the depth of the slots is less than one-quarter wavelength, then the effective surface impedance is dominated by a large positive reactive term and hence its inductance is enhanced above that of a planar metal. Such 
surfaces are thereby able to support $p$-polarized, bound surface waves that are cut off at the resonant frequency, analogous to the limiting surface plasma frequency of metals in the visible regime. The current authors and others have recently explored further resonant subwavelength-structured surfaces that support bound surface waves. ${ }^{13-18}$

Previous studies of diffractively coupled surface waves on gratings at microwave, infrared, or visible frequencies have often been confined to gratings composed of continuous metal. There have been many studies of geometries composed of a periodic array of slits ${ }^{19-24}$ or grooves. ${ }^{11,12,25-32}$ There is also a substantial body of work concerning the enhanced transmission properties of arrays of holes ${ }^{33-42}$ (also Ref. 43 and references therein). These types of arrays support real propagating currents due to the metallic connected nature of their structure. The dispersion of surface modes on these surfaces has also been extensively studied, particularly more recently in the context of enhanced transmission through subwavelength hole arrays ${ }^{35,44-46}$ The surface modes on these square arrays which mediate the transmission are found to be associated with the in-plane diffraction (i.e., the dispersion of the surface modes follow the $k_{g} \hat{k}_{x}$ diffracted light lines) for $p$-(transverse magnetic) polarized incident radiation, and the orthogonal grating wave vector, (i.e., the out-of-plane, $k_{g} \hat{k}_{y}$ diffraction) when $s$-(transverse electric) polarized radiation is incident ${ }^{44}$ There are relatively few studies from the photonics community of the analogous enhanced resonant reflection phenomenon. However it is important to note that the resonant features recorded in the transmission and/or reflection spectra from both connected (holes) and disconnected (patches/particles) arrays, have been associated with excitation of diffractively coupled surface waves, as demonstrated by the work of Minhas et al. ${ }^{47}$ However the electromagnetic response of inductive and capacitive grid frequency selective surfaces (FSSs) away from resonance is well studied ${ }^{48-50}$ but their response is generally not discussed in detail, around the resonance presumably due to the fact that FSSs have only been of interest in the subwavelength regime (i.e., not in the proximity of the onset of diffraction).

In this work, we experimentally record the transmission of a pair of complementary thin arrays of perfectly conducting elements as a function of the polar angle of incidence $\theta$, the frequency $\nu_{0}$, and polarization. Our experimental data illustrate the band structure of the resonances supported, which are attributed to diffractively-coupled surface waves. Of course, for a sample " $a$ " with square symmetry illuminated at normal incidence, Babinet's principle ${ }^{51}$ states that its transmission, $T_{a}$ will be equal to $1-T_{b}$, where $T_{b}$ is the transmission through the complementary structure, $b$. There is a requirement that for oblique incidence, the polarization of the incident radiation is rotated by $90^{\circ}$. Our results validate this application of Babinet's principle in the microwave regime. Furthermore, these experimental results, combined with the predictions of the field solutions from numerical modeling, illustrate that periodicity in the electric and magnetic fields determines the form of the dispersion of these surface modes.
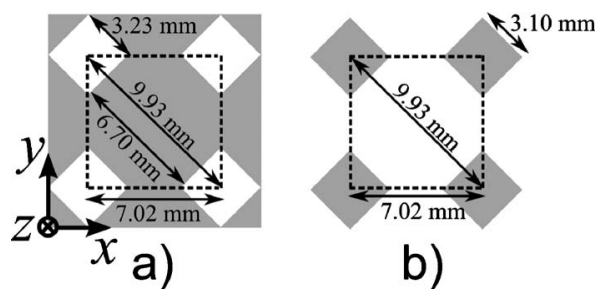

FIG. 1. Schematic representation of the experimental samples. The dotted lines indicate the unit cell of the (a) hole array and (b) disconnected patch array. The shaded area represents aluminum occupancy and the unshaded regions dielectric.

\section{EXPERIMENTAL}

Samples were fabricated by etching a square array (pitch, $\lambda_{g}=7.02 \mathrm{~mm}$ ) of square patches (side length $a$ ), orientated at $45^{\circ}$ with respect to the lattice unit vectors, into aluminised Mylar $^{\circledR}$ (75 $\mu \mathrm{m}$ thickness, $\left.\varepsilon_{\mathrm{r}} \sim 2.6\right)$. The aluminum layer is $\sim 60 \mathrm{~nm}$ thick (determined from scanning electron microscopy measurements), which is much less than the skin depth at the frequency range studied $(\sim 1 \mu \mathrm{m})$. Note however that the layer is almost completely electromagnetically opaque at these wavelengths $(T<1 \%)$ due to the large impedance mismatch presented at the metal-air interface. The $45^{\circ}$ rotated element geometry has been specifically chosen to allow for the study of self-complementary structures. For arrays in which the patch side length is greater than $4.965 \mathrm{~mm}$, neighboring patches overlap, forming a conducting mesh network (a hole array), which is the complementary structure to the patch array.

Figure 1(a) is representative of the connected structure experimentally studied, with square holes of $3.23 \mathrm{~mm}$ side length. Similarly, Fig. 1(b) shows the near-complementary disconnected array, of $3.10 \mathrm{~mm}$ side length patches. The samples were mounted for support on a sheet of expanded polystyrene (refractive index $\sim 1$ ) and placed behind an aperture formed from microwave absorbing material. A collimated microwave beam is incident normal to the surface of the samples. Transmission data for frequencies in the range 26.5-60.0 GHz was obtained by normalizing the transmission through the sample, to that of the aperture and polystyrene sheet alone. The polar angle, $\theta$ in the plane of incidence ( $x z$-plane) was varied from $-60^{\circ}$ to $60^{\circ}$.

Figure 2 shows the normal incidence transmission spectra for both the $3.23 \mathrm{~mm}$ hole array and its near complementary $3.10 \mathrm{~mm}$ patch array structure. Illumination of the connected hole array at normal incidence, reveals a Fano-type resonance giving a transmission peak followed by a transmission minimum is observed on the low frequency side of the onset of first order diffraction. The inverse transmission response is observed for the patch structure, which is, to a good approximation in accordance with Babinet's principle and in agreement with the numerical model. ${ }^{52}$ Note however that the experimental recorded transmission intensity for the hole array does not reach $100 \%$ since there is loss present due to the finite conductivity of the metal. The modeled responses make the assumption that the metal is perfectly conducting and two-dimensional (i.e., zero thickness) and are made to reduce the computation time (the metal thickness is 


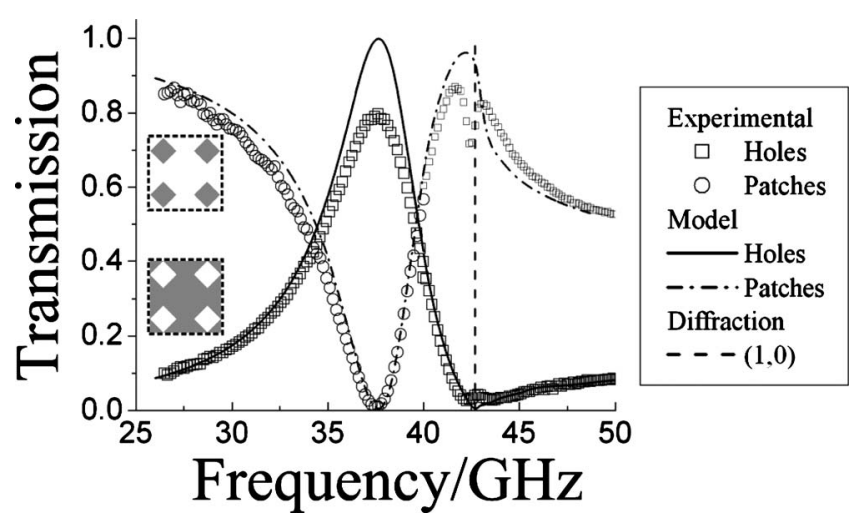

FIG. 2. Zero-order transmission measurements for patches and holes on a square array of pitch $7.02 \mathrm{~mm}$. Normal incidence, electric field vector polarized across the diagonal of the patches. Schematic diagrams illustrate the connectivity of the structure.

very subwavelength $\left(\sim \lambda / 10^{5}\right)$ and would require a very dense number of elements to represent the structure). The permittivity of the Mylar ${ }^{\circledR}$ layer was determined by fitting the microwave transmission peaks of a Mylar ${ }^{\circledR}$ filled slit in metal plates using finite element method (FEM) modeling ${ }^{53}$ and set to $\varepsilon=2.62+0.01 i$ across the frequency range of interest. The experimental data gives good agreement with the model however experimental transmission for the hole array does not reach $100 \%$ since there is loss present due to the finite conductivity of the metal. There is a small incident angle spread of $\sim 1^{\circ}$ inherent in the experimental technique which accounts for the discrepancy between the data and the model near to the diffraction edge where a double peak is observed in the data. This arises from a band gap close to the diffraction edge and the consequential excitation of a mode associated with each band edge. At normal incidence, field symmetry prevents coupling to the upper frequency mode. However at other angles of incidence the symmetry is broken and the incident wave can couple to both the upper and lower branch. Then, because of the finite angle spread in the incident beam, this higher frequency mode is seen for normal incidence resulting in a double peak feature in transmission.

The Fano-type resonance observed in transmission is associated with the excitation of grating-coupled surface waves, as discussed in the literature relating to enhanced transmission through metallic hole arrays. ${ }^{35,39,53}$ Since the Mylar ${ }^{\circledR}$ substrate is very thin $(\ll \lambda)$ the structure can be assumed symmetric. Figure 3 shows the transmission for the $3.23 \mathrm{~mm}$ hole array for both $p$-polarized and $s$-polarized radiation. The intensity of the transmission efficiency is plotted on a logarithmic grayscale to improve clarity of the features close to the onset of diffraction. The transmission responses for $p$-polarized and $s$-polarized radiation at normal incidence $\left(k_{0} \sin \theta=0\right)$ are identical due to the symmetry of the sample.

The angle-dependent transmission data of the hole array structure illuminated with $p$-polarized radiation, shows that the resonant features observed are associated with diffraction from the grating vectors that lie in the plane of incidence. The dispersion of the surface waves strongly follow the inplane diffracted light lines. These straight diffracted light lines arise from the intersection of the light cones centered on $k_{x}= \pm k_{g}$ with the $k_{y}=0$ plane, they intersect at $f=c / \lambda_{g}$

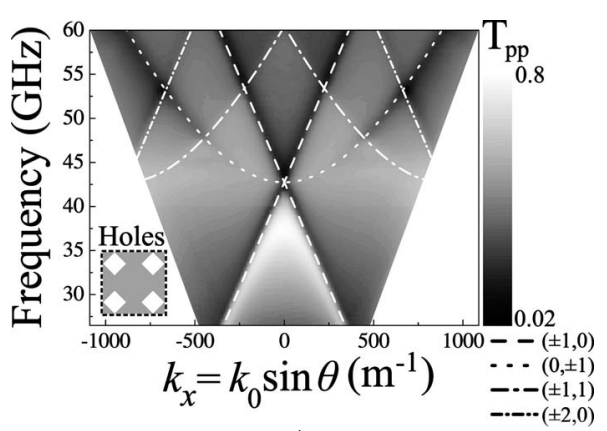

a)

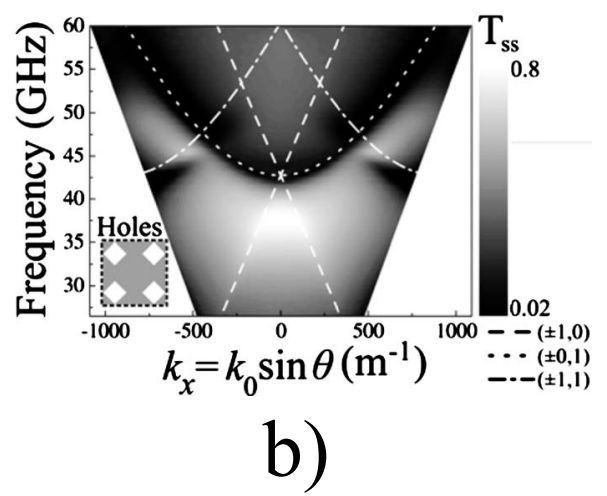

FIG. 3. Zero-order transmission for the $3.23 \mathrm{~mm}$ square hole array sample as a function of in-plane momentum, $k_{x}$, for (a) $p$-polarized and (b) $s$-polarized radiation. Lines indicate sets of diffracted light lines due to grating periodicities. The schematic in the bottom left corner illustrates connectivity of the sample.

$\approx 42.7 \mathrm{GHz}$. There is also evidence of much weaker coupling into the diffracted orders associated with the orthogonal periodicity. Conversely, for $s$-polarized incident radiation [Fig. 3(b)] scattering from the grating vectors that lie out of the plane of incidence is dominant. The surface mode follows the parabolic diffracted light line generated from the intersection of the light cone centered on $k_{y}= \pm k_{g}$ with the $y=0$ plane. This disparity between the coupling efficiency of $p$-polarized and $s$-polarized radiation to the surface modes supported on hole arrays and bigratings has also been reported by other authors. 44,46

Figure 4 shows the dispersion of the resonance for $p$-polarized and $s$-polarized radiation for the $3.10 \mathrm{~mm}$ patch array, the near complementary structure to the $3.23 \mathrm{~mm}$ hole array. For $k_{0} \sin \theta \neq 0$ the dispersion of these modes is reversed in polarization with respect to the response of the complementary structure, i.e., the dispersion of the modes on the patch structure for $p$-polarized incident radiation, are associated with the $\pm k_{g} \hat{\mathbf{k}}_{y}$ diffraction [Fig. 4(a)], are complementary to that of the hole structure for $s$-polarized radiation [Fig. 3(b)], and vice versa. This verifies the requirement of a $90^{\circ}$ rotation of polarization in order to satisfy Babinet's principle. Note that the magnitude of transmission for the pair of experimental structures on resonance is not perfectly complementary since there is absorption inside the metal layer which is not accounted for by Babinet's principle. There are also small differences in sample geometry. Nevertheless, this mode dispersion can be further explained by 


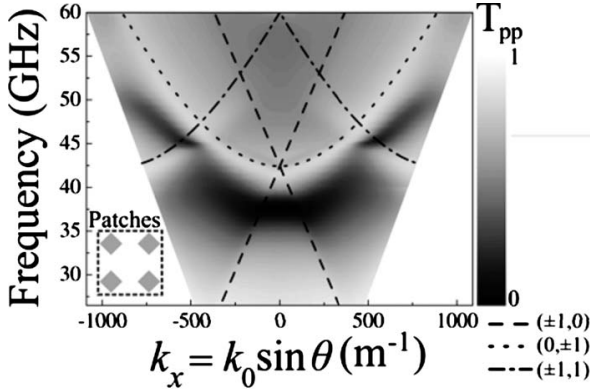

a)

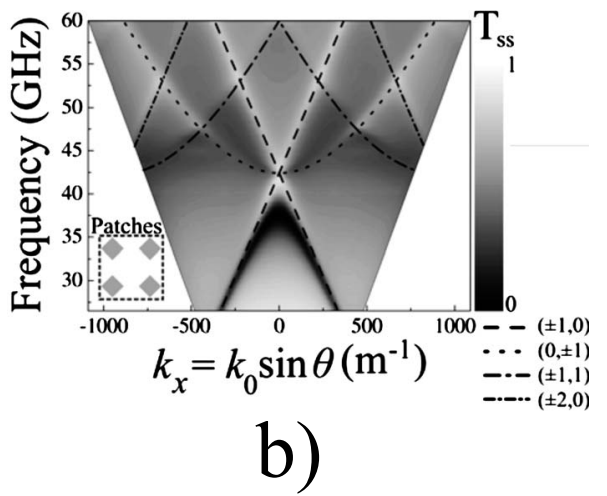

FIG. 4. Zero-order transmission for the $3.10 \mathrm{~mm}$ square patch sample as a function of in-plane momentum, $k_{x}$, for (a) $p$-polarized and (b) $s$-polarized radiation. Lines indicate sets of diffracted light lines due to the grating periodicities. The schematic in the bottom left corner illustrates connectivity of the sample.

examining the resonant fields from FEM modeling (Figs. 5 and 6) using perfect electrical conductor (PEC).

Figure 5 shows the time-averaged magnitude and instantaneous vector surface current density for the $3.23 \mathrm{~mm}$ hole array at a frequency associated with the resonant transmission peak.

The hole array is a connected structure and, therefore, supports real surface currents. The surface currents run parallel to the incident electric field but due to the rotated geometry of the holes, there is enhancement of the surface current density at the narrowest point, i.e., between the corners of the holes at their nearest point. This results in enhanced magnetic field loops in the orthogonal direction to the surface current. Figure 6 shows the time-averaged and instantaneous vector magnetic $(\boldsymbol{H})$ field plot for the $3.23 \mathrm{~mm}$ hole array on resonance. Again the fields are plotted at a phase corresponding to maximum field enhancement.

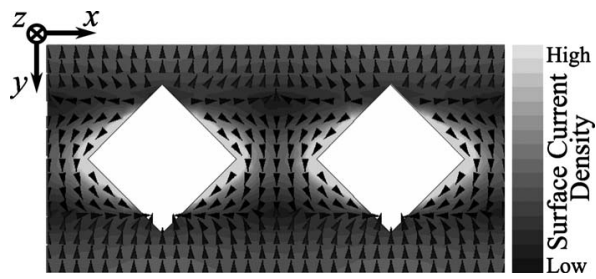

FIG. 5. Surface current density magnitude plotted on the surface of the PEC hole array on resonance $(37.6 \mathrm{GHz})$ for two unit cells, $\theta=0^{\circ}$. The incident electric field is polarized along the $y$-axis. Arrows represent the direction of the surface current at a phase corresponding to maximum surface current density.

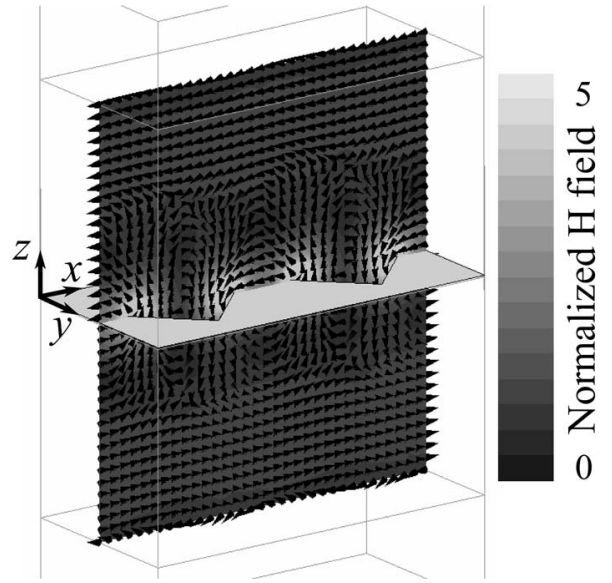

FIG. 6. Time-averaged magnetic field enhancement plotted on resonance $(37.6 \mathrm{GHz})$ through the center of two holes with arrows representing the direction of the field at a phase corresponding to maximum field enhancement, $\theta=0^{\circ}$. Gray represents the metal. The incident electric field is polarized along the $y$-axis.

Magnetic field loops are present between neighboring holes that lie in the $x z$-plane, i.e., parallel to the incident magnetic field. The dominant periodicity of the structure that influences the dispersion of the surface mode can be determined by examining the fields away from the sample in the transmitted half space. This allows the transmitted fields to be considered without the influence of near-field evanescent effects close to the structure. Figure 7 shows the timeaveraged magnetic fields on resonance parallel to the incident magnetic vector at a distance $3 \mathrm{~mm}$ below the surface of the sample.

The magnetic fields show periodicity in the direction of the incident electric field ( $y$-direction) resulting in dominant scattering from the $k_{g} \hat{k}_{y}$ grating vector. Since the coupling efficiency into these diffracted orders is most dominant the surface wave disperses along the parabolic out-of-plane diffracted light lines [Fig. 3(b)] for nonzero angles of incidence. A similar description can be used to describe the response for the orthogonal polarization with the scattering from the $k_{g} \hat{k}_{x}$ grating vector leading to the linear dispersion seen in Fig. 3(a).

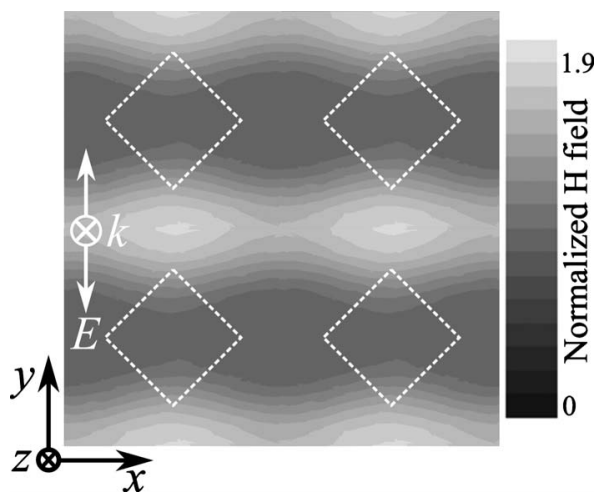

FIG. 7. Time-averaged resonant magnetic fields on resonance $(37.6 \mathrm{GHz})$ parallel to the direction of the incident magnetic vector (in the $x$-direction) 3 $\mathrm{mm}$ below the hole array, $\theta=0^{\circ}$. The field is plotted across four unit cells for clarity. White dashed lines indicate the position of the holes. 


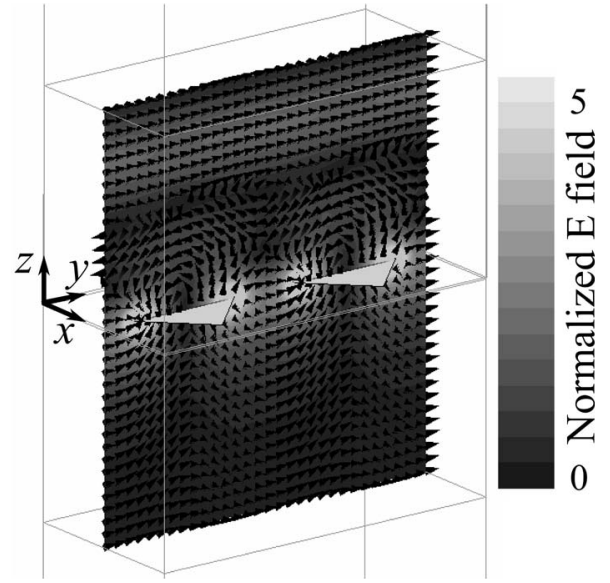

FIG. 8. Time-averaged electric field enhancement on resonance $(37.4 \mathrm{GHz})$ plotted through the center of two patches with arrows representing the direction of the field at a phase corresponding to maximum field enhancement, $\theta=0^{\circ}$. Gray represents the metal. The incident electric field vector is polarized along the $y$-axis.

Field plots from the finite element method modeling for the $3.10 \mathrm{~mm}$ patch array are plotted in Fig. 8 .

On resonance loops of electric field are present between neighboring patches in the $y z$-plane, i.e., in the direction of the incident electric field. Again the dominant periodicity can be determined by examining the fields below the surface of the sample. Figure 9 shows the time-averaged electric fields on resonance parallel to the incident electric vector $8 \mathrm{~mm}$ below the surface of the sample.

The electric fields show periodicity in the direction orthogonal to the incident electric field (y-direction) resulting in dominant scattering from the $k_{g} \hat{k}_{x}$ grating vector. Since the coupling efficiency into these diffracted orders is most dominant the surface wave disperses along the linear in-plane diffracted light lines [Fig. 4(b)]. A similar description can be used to explain the response for the orthogonal polarization with the scattering from the $k_{g} \hat{k}_{y}$ grating vector leading to the parabolic dispersion seen in Fig. 4(a).

The band structures of surface wave resonances on disconnected arrays are clearly reversed with respect to their polarization response from those observed on a grating of continuous metal..$^{35,44-46}$ This interchange in behavior of the

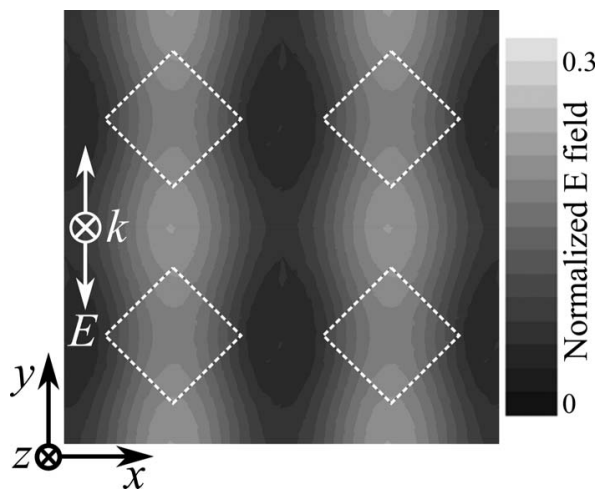

FIG. 9. Time-averaged resonant electric fields on resonance $(37.4 \mathrm{GHz})$ parallel to the direction of the incident electric vector (in the $y$-direction) 8 $\mathrm{mm}$ below the patch array, $\theta=0^{\circ}$. Fields plotted across four unit cells for clarity. White dashed lines indicate the position of the patches. electric and magnetic fields is, however, completely predicted by Babinet's principle to describe the nature of complementary structures.

\section{CONCLUSION}

In summary, the dispersion of surface modes on two near complementary thin metallic gratings in the microwave regime have been studied, showing the well known enhanced transmission, or the corresponding, lesser studied, enhanced reflection phenomena. The modes for a given linear polarization are found to closely follow the light lines associated with $\pm k_{g} \hat{\mathbf{k}}_{x}$ or $\pm k_{g} \hat{\mathbf{k}}_{y}$ diffraction, depending on the connectivity of the structure, with a near identical dispersion being observed for the array's complementary structure if the polarization of the incident microwaves is rotated by $90^{\circ}$. The results accord well with the electromagnetic form of Babinet's principle. FEM modeling shows that for the disconnected patch arrays, strong electric fields are present between neighboring patches and there is strong modulation in the electric field perpendicular to the plane of incidence. By contrast the holes in the complementary structure have strong loops of magnetic field between them with strong modulation in the magnetic field in the plane of incidence.

\section{ACKNOWLEDGMENTS}

JDE acknowledges the support of DSTL and EPSRC through an industrial CASE studentship. APH thanks the EPSRC for continued financial support through his Advanced Research Fellowship (grant no. GR/S96029/01). CCrown copyright 2009. This work is part funded by the Ministry of Defense and is published with the permission of the Defense Science and Technology Laboratory on behalf of the Controller of HMSO.

${ }^{1}$ J. Zenneck, Ann. Phys. 28, 665 (1909).

${ }^{2}$ A. Sommerfeld, Ann. Phys. Chem. 67, 233 (1899).

${ }^{3}$ A. Sommerfeld, Ann. Phys. 333, 665 (1909).

${ }^{4}$ F. Yang, J. R. Sambles, and G. W. Bradberry, Phys. Rev. B 44, 5855 (1991).

${ }^{5}$ U. Fano, Phys. Rev. 118, 451 (1960).

${ }^{6}$ J. Zenneck, Ann. Phys. 328, 846 (1907).

${ }^{7}$ H. Raether, Surface Plasmons on Smooth and Rough Surfaces and on Gratings (Springer, Berlin, 1988).

${ }^{8}$ R. W. Ziolkowski and E. Heyman, Phys. Rev. E 64, 056625 (2001).

${ }^{9}$ E. Kretschmann and H. Raether, Z. Naturforsch. A 23, 2135 (1968).

${ }^{10}$ A. Otto, Z. Phys. 216, 398 (1968).

${ }^{11}$ H. M. Barlow and A. L. Cullen, Proc. Inst. Electr. Eng. 100, 329 (1953).

${ }^{12}$ C. C. Cutler, IEEE Antennas and Propagation Society International Symposium 3, 1456 (1994)

${ }^{13}$ J. B. Pendry, L. Martín-Moreno, and F. J. García-Vidal, Science 305, 847 (2004).

${ }^{14}$ A. P. Hibbins, B. R. Evans, and J. R. Sambles, Science 308, 670 (2005).

${ }^{15}$ S. A. Maier, S. R. Andrews, L. Martín-Moreno, and F. J. Garcia-Vidal, Phys. Rev. Lett. 97, 176805 (2006).

${ }^{16}$ Q. Gan, Z. Fu, Y. J. Ding, and F. J. Bartoli, Phys. Rev. Lett. 100, 256803 (2008).

${ }^{17}$ D. Sievenpiper, L. Zhang, R. F. J. Broas, N. G. Alexopolous, and E. Yabolonovitch, IEEE Trans. Microwave Theory Tech. 47, 2059 (1999).

${ }^{18}$ M. J. Lockyear, A. P. Hibbins, and J. R. Sambles, Phys. Rev. Lett. 102, 073901 (2009)

${ }^{19}$ C. H. Palmer, J. Opt. Soc. Am. 42, 269 (1952).

${ }^{20}$ U. Schröter and D. Heitmann, Phys. Rev. B 58, 15419 (1998).

${ }^{21}$ J. A. Porto, F. J. García-Vidal, and J. B. Pendry, Phys. Rev. Lett. 83, 2845 (1999). 
${ }^{22}$ H. E. Went, A. P. Hibbins, J. R. Sambles, C. R. Lawrence, and A. P. Crick, Appl. Phys. Lett. 77, 2789 (2000).

${ }^{23}$ S. Astilean, P. Lalanne, and M. Palamaru, Opt. Commun. 175, 265 (2000).

${ }^{24}$ S. Collin, G. Vincent, R. Haïdar, N. Bardou, S. Rommeluère, and J. L. Pelouard, Phys. Rev. Lett. 104, 027401 (2010).

${ }^{25}$ R. W. Wood, Philos. Mag. 4, 396 (1902).

${ }^{26}$ U. Fano, J. Opt. Soc. Am. 31, 213 (1941).

${ }^{27}$ W. C. Meecham and C. W. Peters, J. Appl. Phys. 28, 216 (1957).

${ }^{28}$ C. H. Palmer, F. C. Evering, and F. M. Nelson, Appl. Opt. 4, 1271 (1965).

${ }^{29}$ J. R. Andrewartha, J. R. Fox, and I. J. Wilson, Opt. Acta 26, 197 (1979).

${ }^{30}$ E. Popov, L. Tsonev, and D. Maystre, Appl. Opt. 33, 5214 (1994).

${ }^{31}$ A. P. Hibbins, J. R. Sambles, and C. R. Lawrence, J. Appl. Phys. 86, 1791 (1999).

${ }^{32}$ E. Popov, D. Maystre, R. C. McPhedran, M. Nevière, M. C. Hutley, and G. H. Derrick, Opt. Express 16, 6146 (2008).

${ }^{33}$ C.-C. Chen, IEEE Trans. Microwave Theory Tech. 19, 475 (1971).

${ }^{34}$ R. Ulrich and M. Tacke, Appl. Phys. Lett. 22, 251 (1973).

${ }^{35}$ T. W. Ebbesen, H. J. Lezec, H. F. Ghaemi, T. Thio, and P. A. Wolff, Nature (London) 391, 667 (1998).

${ }^{36}$ T. Thio, H. F. Ghaemi, H. J. Lezec, P. A. Wolff, and T. W. Ebbesen, J. Opt. Soc. Am. B 16, 1743 (1999).

${ }^{37}$ E. Popov, M. Nevière, S. Enoch, and R. Reinisch, Phys. Rev. B 62, 16100 (2000).

${ }^{38}$ A. Krishnan, T. Thio, T. J. Kim, H. J. Lezec, T. W. Ebbesen, P. A. Wolff, J. Pendry, L. Martin-Moreno, and F. J. Garcia-Vidal, Opt. Commun. 200,
1 (2001).

${ }^{39}$ L. Martín-Moreno, F. J. García-Vidal, H. J. Lezec, K. M. Pellerin, T. Thio, J. B. Pendry, and T. W. Ebbesen, Phys. Rev. Lett. 86, 1114 (2001).

${ }^{40}$ M. Beruete, M. Sorolla, I. Campillo, J. S. Dolado, L. Martín Moreno, J. Bravo Abad, and F. J. García Vidal, Opt. Lett. 29, 2500 (2004).

${ }^{41}$ B. Hou, Z. H. Hang, W. J. Wen, C. T. Chan, and P. Sheng, Appl. Phys. Lett. 89, 131917 (2006).

${ }^{42}$ J. Braun, B. Gompf, G. Kobiela, and M. Dressel, Phys. Rev. Lett. 103, 203901 (2009).

${ }^{43}$ F. J. García de Abajo, Rev. Mod. Phys. 79, 1267 (2007).

${ }^{44}$ W. L. Barnes, W. A. Murray, J. Dintinger, E. Devaux, and T. W. Ebbesen, Phys. Rev. Lett. 92, 107401 (2004).

${ }^{45}$ H. F. Ghaemi, T. Thio, D. E. Grupp, T. W. Ebbesen, and H. J. Lezec, Phys. Rev. B 58, 6779 (1998).

${ }^{46}$ S. Collin, C. Sauvan, C. Billaudeau, F. Pardo, J. C. Rodier, J. L. Pelouard, and P. Lalanne, Phys. Rev. B 79, 165405 (2009).

${ }^{47}$ B. K. Minhas, W. Fan, K. Agi, S. R. J. Brueck, and K. J. Malloy, J. Opt. Soc. Am. A Opt. Image Sci. Vis 19, 1352 (2002).

${ }^{48}$ R. Ulrich, Infrared Phys. 7, 37 (1967).

${ }^{49}$ S. T. Shanahan and N. R. Heckenberg, Appl. Opt. 20, 4019 (1981).

${ }^{50}$ R. Ulrich, Appl. Opt. 7, 1987 (1968).

${ }^{51}$ M. Babinet, Comptes Rendus de l'Académie des Sciences 4, 638 (1837).

${ }^{52}$ HFSS, Ansoft Corporation, Pittsburgh, PA, USA.

${ }^{53}$ A. P. Hibbins, M. J. Lockyear, I. R. Hooper, and J. R. Sambles, Phys. Rev. Lett. 96, 073904 (2006). 